\title{
Synthesis of Putrescine Bisamides as Antimicrobial and Anti-Inflammatory Agents
}

\author{
Srinuvasarao Rayavarapu ${ }^{1}$, Sunanda Kumari Kadiri², Mahaboob Basha Gajula', Mangarao Nakka1, Ramu Tadikonda, Nagendra Sastry \\ Yarla $^{3}$ and Siddaiah Vidavalur ${ }^{1 *}$
}

${ }^{1}$ Department of Organic Chemistry \& FDW, Andhra University, Visakhapatnam, India

${ }^{2}$ Department of Microbiology, Andhra University, Visakhapatnam, India

${ }^{3}$ Department of Biotechnology, Gitam University, Visakhapatnam, India

\begin{abstract}
A new naturally occurring $N_{1}, N_{6}$-dihydrocinnamyl putrescine bisamide, JBIR-94, along with nine structural analogs and a series of substituted phenyl and alkyl putrescine bisamides have been synthesized from putrescine and appropriately substituted carboxylic acids, through carboxylic acid chlorides. Antimicrobial, 5-Lipooxygenase enzyme inhibitory and antioxidant studies were performed for all synthesized compounds. Dihydrocinnamyl series of putrescine bisamides (4a-4i) showed good bioactivities compared to substituted phenyl (6a-6g) and diakyl (6h-6j) series of compounds. Among all compounds, 4h (methylenedioxy analog) and 4a (JBIR-94) showed good antimicrobial, antiinflammatory and antioxidant activities.
\end{abstract}

Keywords: JBIR-94; Putrescine bisamide; Antimicrobial; Antioxidant; 5-LOX; Docking

\section{Introduction}

Putrescine bisamides are one of the subclasses of naturally occurring polyamides [1]. During the last two decades several symmetrical and unsymmetrical putrescine bisamides have been isolated from Aglaia [2-4], Liberica [5], and Carydalis [6], species. These compounds have been reported to possess various biological activities including cytotoxicity [7], anti-inflammatory [8], antioxidant $[9,10]$, insecticidal [11,12], and antiviral activity. Putrescine derivatives have been hypothesized to be one of the precursors in bio-synthesis of rocaglamides, which have displayed pronounced anti proliferative activity against Human cancer cell $[10,13]$, and exhibit strong insecticidal activity against Spodoptera littoralis [13]. The insecticidal activity of rocaglamides is comparable to the potency of azadirachtin [13]. Synthesis of these compounds is usually based on the condensation of putrescine with carboxylic acid chlorides in the presence of bases, or with carboxylic acids in the presence of coupling reagents such as 1-Ethyl-3-(3-dimethylaminopropyl) carodiimide (EDC), $N, N^{\prime}$-Dicyclohexylcarbodiimide (DCC) and (Benzotriazol-1yloxy)tris(dimethylamino) phosphonium hexaflurophosphate (BOP). Recently, a new $N_{1}, N_{6}$-dihydrocinnamyl putrescine bisamide, JBIR-94, has been isolated from broth culture of Streptomyces (strain R56-07), and reported to have antioxidant activities [9]

\section{Experimental Section}

\section{General}

Melting points were recorded on a Mel-Temp melting point apparatus, in open capillaries and are uncorrected. ${ }^{1} \mathrm{H}$ NMR $(400 \mathrm{MHz})$, ${ }^{13} \mathrm{C}$ NMR $(100 \mathrm{MHz})$ spectra were recorded on a Bruker AMX $400 \mathrm{MHz}$ NMR spectrometer using TMS as internal standard and the values for chemical shifts $(\delta)$ being given in ppm and coupling constants $(J)$ in Hertz (Hz). Mass spectra were recorded on an Agilent 1100 LC/MSD. Acme silica gel G and silica gel (100-200 mesh) were used for analytical TLC and column chromatography, respectively. Other chemicals were purchased from Sigma Aldrich and used without further purification.

General experimental procedure for synthesis of 3: A mixture of carboxylic acid $(6.58 \mathrm{mmol})$ and thionyl chloride $(13.1 \mathrm{mmol})$ was refluxed at $80^{\circ} \mathrm{C}$ for $1 \mathrm{~h}$. After $1 \mathrm{~h}$ excess of $\mathrm{SOCl}_{2}$ was removed under reduced pressure. Triethylamine $(14.2 \mathrm{mmol})$ and putrescine $(3.3$ mmol) dissolved in DCM was added to the above reaction mixture at $0^{\circ} \mathrm{C}$ and warm to room temperature for $1 \mathrm{~h}$. Then the solution was diluted with $\mathrm{CHCl}_{3}$ and washed consequently with $2 \mathrm{~N} \mathrm{HCl}$, saturated aq $\mathrm{NaHCO}_{3}$ and brine solution. The organic phase was dried over $\mathrm{Na}_{2} \mathrm{SO}_{4}$ and the solvent was removed under vacuum. The residue was suspended in ethyl acetate filtered off and washed with cold ethyl acetate and dried to obtain the pure product. It was directly used in the next step.

General experimental procedure for synthesis of 4: The compound ( $0.5 \mathrm{gm})$ (3) was dissolved in THF, and $10 \%$ palladium on carbon $(5 \mathrm{mg}, 10 \%)$ was added. The mixture was stirred under hydrogen balloon pressure at ambient temperature for $2 \mathrm{~h}$. The reaction mixture was filtered through celite bed and washed with methanol. Methanol was evaporated under reduced pressure and the resultant solid was recrystallized in cold ethyl acetate to afford title compound.

General experimental procedure for synthesis of 6: Putrescine $(3.3 \mathrm{mmol})$ and triethylamine $(14.3 \mathrm{mmol})$ were added to the solution of acid chloride $(7.6 \mathrm{mmol})$ in $\mathrm{CH}_{2} \mathrm{Cl}_{2}$ at $0^{\circ} \mathrm{C}$. The reaction mixture was then allowed to stir at room temperature for $0.5 \mathrm{~h}$. After the completion of the reaction mixture was basicified with saturated aq $\mathrm{NaHCO}_{3}$ solution and extracted with $\mathrm{CH}_{2} \mathrm{Cl}_{2}$. The organic layer was dried over anhydrous $\mathrm{Na}_{2} \mathrm{SO}_{4}$ and concentrated under reduced pressure to afford 6. This was purified by recrystallization with hexane.

\section{Antimicrobial assay}

Antimicrobial assay performed on human pathogenic bacteria and fungi. Salmonella typhi, Vibrio cholerae, Shigella dysenteriae, Enterococcus faecalis are gastrointestinal pathogenic bacteria, which

*Corresponding author: Siddaiah Vidavalur, Department of Organic Chemistry \& FDW, Andhra University, Visakhapatnam-530003, India, Tel: 09440754164 E-mail: sidduchem@gmail.com

Received February 14, 2014; Accepted March 22, 2014; Published March 24 2014

Citation: Rayavarapu S, Kadiri SK, Gajula MB, Nakka M, Tadikonda R, et al (2014) Synthesis of Putrescine Bisamides as Antimicrobial and Anti-Inflammatory Agents. Med chem 4: 367-372. doi:10.4172/2161-0444.1000167

Copyright: ( 2014 Rayavarapu S, et al. This is an open-access article distributed under the terms of the Creative Commons Attribution License, which permits unrestricted use, distribution, and reproduction in any medium, provided the original author and source are credited. 
are clinical isolates collected from King Gerorge Govt. Hospital, Visakhapatnam, India. Staphylococcus aureus (NCIM 3021) culture was purchased at NCL, Pune, India. Candida albicans is a dermatophytic fungus collected from K. Ramamurthy memorial hospital, Ravivalsa, India. Zone of inhibitions were determined using agar well diffusion method and minimum inhibitory concentration (MIC) was done by broth dilution assay. Microbial broth cultures (Mueller Hinton broth for bacteria, Sabouraud Dextrose broth for fungi) were adjusted to an absorbance of 0.6 (Optical Density at $620 \mathrm{~nm}$ ) in Spectrophotometer according to CLSI guidelines. These cultures were used as Inoculums for antimicrobial study. The agar plates were prepared by pour plate method using $20 \mathrm{ml}$ of sterilized agar medium ( $\mathrm{MH}$ agar for bacteria, $\mathrm{SD}$ agar for fungi). The sterile agar medium was cooled to $45^{\circ} \mathrm{C}$ and mixed thoroughly with $1 \mathrm{ml}$ of growth culture of concerned test organism (inoculum) and then poured into the sterile petri dishes and allowed to solidify. Wells of $6 \mathrm{~mm}$ size were made with sterile cork borer and test compounds were added. The agar plates were incubated at for 4 days at $28^{\circ} \mathrm{C}$ for fungi wile 24 hours at $37^{\circ} \mathrm{C}$ for bacteria. Zone of inhibitions were measured by Himedia milli meter zone reader. Minimum Inhibitory Concentration (MIC) was performed on broth media $(10 \mathrm{ml})$ containing $1000-1 \mu \mathrm{g} / \mathrm{ml}$ of test compound prepared by 10 fold dilution. $0.1 \mathrm{ml}$ of culture inoculums was added. The MIC was determined at the concentration of compound that causes nil absorbance (no growth) in the spectrophotometer at $620 \mathrm{~nm}$. All the experiments were conducted according to Clinical Laboratory Standard Institute. Ciprofloxacin (for bacteria) and Griseofulvin (for fungi) were used as positive control. DMSO used as negative control [14-18].

\section{5-Lipoxygenase (5-LOX) inhibitory assay}

5-LOX from potato tubers was purified and assayed as per the method described by Reddanna et al. [18] The assay mixture contained $80 \mathrm{mM}$ linoleic acid and sufficient amount of potato 5-Lipoxygenase enzyme in $50 \mathrm{mM}$ phosphate buffer $(\mathrm{pH}$ 6.3). The reaction was initiated by the addition of enzyme buffer mix to substrate (linoleic acid) and the enzyme activity was monitored by an increase in absorbance at $234 \mathrm{~nm}$ for 120 seconds using UV Kinetic mode on Varian Cary-50 UV-VIS spectrophotometer. In the inhibition studies the activities were measured by incubating various concentration of compound with enzyme buffer mix for two minutes before adding the substrate. The assay was performed in triplicate. Percentage of inhibition was calculated by change in absorbance of test with that of control enzyme activity. Nordihydroguaiaretic acid (NDGA) was used as positive control [19-21].

\section{Molecular docking studies}

5-Lipoxygenase (PDB ID 308Y) X-Ray crystal structure was obtained from Protein Data Bank and used in docking studies. Cocystalized ligands and water molecules are removed from target protein using Argus lab. Ligands are prepared using Chemoffice (Cambridge). Energy minimization was done using molecular mechanics. The minimized was executed until root mean square value reached smaller than $0.001 \mathrm{Kcal} / \mathrm{mol}$. Such energy minimized ligands and receptor used for docking studies using GEMDOCK (Generic Evolutionary Method for molecular DOCKing) is a generic evolutionary method with an empirical scoring function for the protein-ligand docking, which is a problem of paramount importance in structure-based drug design, combines both continuous and discrete search mechanisms. A population size of 300 with 70 generations and 3 solutions were used in docking accuracy setting. PyMol is used for better visualization of interactions [22-24].

\section{DPPH radical scavenging activity}

DPPH (1, 1-diphenyl -2-picryl -hydrazyl) radical scavenging activity of the compounds was determined by the method of Lamaison et al., [19] which depends on scavenging of coloured free radical $(\mathrm{DPPH})$ in methanol solution by test compound. The reaction mixture contains DPPH and compound in a final concentration in $3 \mathrm{ml}$. Absorption of DPPH at its absorption maximum $516 \mathrm{~nm}$ is inversely proportional to scavenging activity of compound. The activity was expressed as inhibitory concentration $50\left(\mathrm{IC}_{50}\right)$ i.e. the concentration of test compound required to give $50 \%$ reduction in absorbance of test solution compared to that of blank solution [25].

\section{Superoxide scavenging activity}

Superoxide scavenging activity of the synthesized compounds was determined by the method of Mc Cord \& Fridovich [20] (1969), modified by Ruby et al. [21], which depends on the light induced superoxide generation by riboflavin and the corresponding reduction of NBT. The assay mixture contained different concentrations of the test substances and EDTA ( $6 \mathrm{mM}$ containing $3 \mu \mathrm{g} \mathrm{NaCN})$, NBT $(5 \mu \mathrm{M})$ and phosphate buffer $58 \mathrm{mM}, \mathrm{pH} 7.8$ ) in a total vol. of $300 \mu \mathrm{l}$. The wells received uniform illumination for $15 \mathrm{~min}$ and thereafter optical density was measured at $560 \mathrm{~nm}[26,27]$.

In conclusion, a new naturally occurring $N_{1}, N_{6}$-didihydrocinnamyl putrescine bisamide, JBIR-94, along with nine structural analogs and a series of substituted phenyl and alkyl putrescine bisamides have been synthesized from putrescine and appropriately substituted carboxylic acids, through carboxylic acid chlorides. Antimicrobial, 5-LOX enzyme inhibitory and antioxidant studies were performed for all synthesized compounds. Dihydrocinnamyl series of putrescine bisamides (4a4i) showed efficient bioactivities compared to substituted phenyl $(6 a-6 g)$ and dialkyl (6h-6j) series of compounds. Among the tested compounds, 4a (JBIR-94) and $4 \mathrm{~h}$ showed potent antimicrobial, and anti-inflammatory activities $[28,29]$.

\section{Characterization of putrescine bisamides}

N, $\quad N^{\prime}$-(Butane-1,4-diyl)bis(3-(4-hydroxy-3-methoxyphenyl) propanamide) (4a). Gray solid, yield $90 \%, \mathrm{mp} 140-148^{\circ} \mathrm{C} ;{ }^{1} \mathrm{H}$ NMR $(400 \mathrm{MHz}, \mathrm{MeOD}) \delta 6.67(\mathrm{~d}, J=1.6 \mathrm{~Hz}, 2 \mathrm{H}), 6.59(\mathrm{~d}, J=8 \mathrm{~Hz}, 2 \mathrm{H})$, $6.52(\mathrm{dd}, J=1.6,8.0 \mathrm{~Hz}, 2 \mathrm{H}), 3.72(\mathrm{~s}, 6 \mathrm{H}), 2.97$ (brs, $4 \mathrm{H}), 2.71(\mathrm{t}, J=$ $7.6 \mathrm{~Hz}, 4 \mathrm{H}), 2.32(\mathrm{t}, J=7.6 \mathrm{~Hz}, 4 \mathrm{H}), 1.18(\mathrm{~m}, 4 \mathrm{H}) ;{ }^{13} \mathrm{C}$ NMR $(100 \mathrm{MHz}$, MeOD) $\delta 175.4,148.9,145.9,133.8,121.9,116.3,113.4,56.5,39.9,39.4$, 32.6, 27.6; LC-MS: $m / z$ : 443.3 ( M-H ); Anal. Calcd. for $\mathrm{C}_{24} \mathrm{H}_{52} \mathrm{~N}_{2} \mathrm{O}_{6}$; C, 64.85; H, 7.26, N, 6.30 Found: C, 64.80; H, 7.30, N, 6.25.

$N, N^{\prime}$-(Butane-1,4-diyl) bis (3-(3-methoxyphenyl) propanamide) (4b). White solid, yield $92 \%$, mp $160-166^{\circ} \mathrm{C} ;{ }^{1} \mathrm{H}$ NMR $(400 \mathrm{MHz}$, MeOD) $\delta 7.05(\mathrm{t}, J=8 \mathrm{~Hz}, 2 \mathrm{H}), 6.67(\mathrm{~m}, 4 \mathrm{H}), 6.62(\mathrm{dd}, J=1.2,8.0 \mathrm{~Hz}$, $2 \mathrm{H}), 3.65(\mathrm{~s}, 6 \mathrm{H}), 2.98(\mathrm{t}, J=6.4 \mathrm{~Hz}, 4 \mathrm{H}), 2.76(\mathrm{t}, J=7.6 \mathrm{~Hz}, 4 \mathrm{H}), 2.35$ $(\mathrm{t}, J=7.6 \mathrm{~Hz}, 4 \mathrm{H}), 1.22$ (brs, $4 \mathrm{H}) ;{ }^{13} \mathrm{C}-\mathrm{NMR}(100 \mathrm{MHz}, \mathrm{MeOD}) \delta 175.1$, $161.3,143.7,130.4,121.8,115.2,112.6,55.6,39.9,38.9,32.9,27.6$; LCMS: $m / z 411.3$ ( M-H ); Anal. Calcd. for. $\mathrm{C}_{24} \mathrm{H}_{32} \mathrm{~N}_{2} \mathrm{O}_{4} \mathrm{C}, 69.88 ; \mathrm{H}, 7.82$; N, 6.79; Found: C, 69.80; H, 7.886; N, 6.82 .

$N, N^{\prime}$-(Butane-1,4-diyl)bis(3-(2-methoxyphenyl)propanamide) (4c). White solid, yield $92 \%, \mathrm{mp} 150-156^{\circ} \mathrm{C},{ }^{1} \mathrm{H}$ NMR $(400 \mathrm{MHz}$, DMSO- $\left.d_{6}\right) \delta 7.73(\mathrm{t}, J=4.8 \mathrm{~Hz}, 2 \mathrm{H}), 7.17(\mathrm{t}, J=7.6 \mathrm{~Hz}, 2 \mathrm{H}), 7.12(\mathrm{~d}$, $J=7.2 \mathrm{~Hz}, 2 \mathrm{H}) 6.93(\mathrm{~d}, J=8.0 \mathrm{~Hz}, 2 \mathrm{H}), 6.84(\mathrm{t}, J=7.2 \mathrm{~Hz}, 2 \mathrm{H}), 3.78$ (s, $6 \mathrm{H}), 3.01(\mathrm{~d}, J=4.8 \mathrm{~Hz}, 4 \mathrm{H}), 2.76(\mathrm{t}, J=7.6 \mathrm{~Hz}, 4 \mathrm{H}), 2.30(\mathrm{t}, J=8.0$ $\mathrm{Hz}, 4 \mathrm{H}), 1.33$ (brs ,4H); ${ }^{13} \mathrm{C}$ NMR (100 MHz DMSO- $\left.d_{6}\right) \delta 171.3,156.9$, 129.3 , 129.1, 127.2, 120.2, 110.5, 55.2, 38.1, 35.4, 26.6, 25.7; LC-MS: $m / z$ 
$413.5(\mathrm{M}+\mathrm{H})^{+}, 435.4(\mathrm{M}+\mathrm{Na})^{+}$; Anal. Calcd. for $\mathrm{C}_{24} \mathrm{H}_{32} \mathrm{~N}_{2} \mathrm{O}_{4}, \mathrm{C}, 69.88$; H, 7.82; N, 6.79; Found: C, 69.78; H, 7.88; N, 6.80 .

$N, N^{\prime}$-(Butane-1,4-diyl)bis(3-(4-methoxyphenyl)propanamide) (4d). White solid, yield $92 \%, \mathrm{mp} 158-162^{\circ} \mathrm{C},{ }^{1} \mathrm{H}$ NMR $(400 \mathrm{MHz}$, DMSO- $\left.d_{6}\right) \delta 8.01(\mathrm{t}, J=5.2 \mathrm{~Hz}, 2 \mathrm{H}), 7.49(\mathrm{~d}, J=8.4 \mathrm{~Hz}, 4 \mathrm{H}), 6.96(\mathrm{~d}, J=$ $8.4 \mathrm{~Hz}, 4 \mathrm{H}), 3.78(\mathrm{~s}, 6 \mathrm{H}), 2.98(\mathrm{t}, J=6.4 \mathrm{~Hz}, 4 \mathrm{H}), 2.75(\mathrm{t}, J=7.6 \mathrm{~Hz}, 4 \mathrm{H})$, $2.36(\mathrm{t}, J=7.6 \mathrm{~Hz}, 4 \mathrm{H}), 1.22$ (brs, $4 \mathrm{H}) .{ }^{13} \mathrm{C}$ NMR $\left(100 \mathrm{MHz}, \mathrm{DMSO}-d_{6}\right)$ $\delta 171.2,156.9,129.3,129.1,127.2,120.2,110.5,53.2,38.9,38.1,35.4$, 25.6, 25.7; LC-MS: $m / z 413.5(\mathrm{M}+\mathrm{H})^{+}, 435.4(\mathrm{M}+\mathrm{Na})^{+}$. Anal. Calcd for. $\mathrm{C}_{24} \mathrm{H}_{32} \mathrm{~N}_{2} \mathrm{O}_{4}, \mathrm{C}, 69.88 ; \mathrm{H}, 7.82 ; \mathrm{N}, 6.79$; Found: C, 69.83; H, 7.87; $\mathrm{N}, 6.83$.

$N, N^{\prime}$-(Butane-1,4-diyl)bis (3-(3,4-dimethoxyphenyl) propanamide (4e). White solid, yield $90 \%, \mathrm{mp} 176-178^{\circ} \mathrm{C}{ }^{1} \mathrm{H}$ NMR $(400 \mathrm{MHz}, \mathrm{MeOD}) \delta 6.74(\mathrm{~d}, J=8.4 \mathrm{~Hz}, 2 \mathrm{H}), 6.71(\mathrm{~s}, 2 \mathrm{H}), 6.64(\mathrm{~d}, J=$ $8.0 \mathrm{~Hz}, 2 \mathrm{H}), 3.70(\mathrm{~s}, 6 \mathrm{H}), 3.68(\mathrm{~s}, 6 \mathrm{H}), 2.97(\mathrm{t}, J=6.0 \mathrm{~Hz}, 4 \mathrm{H}), 2.74(\mathrm{t}, J$ $=7.6 \mathrm{~Hz}, 4 \mathrm{H}), 2.34(\mathrm{t}, J=7.6 \mathrm{~Hz}, 4 \mathrm{H}), 1.20(\mathrm{t}$, brs, $4 \mathrm{H}) ;{ }^{13} \mathrm{C}$ NMR $(100$ $\mathrm{MHz}, \mathrm{MeOD}) \delta 175.2,150.5,149.1,135.3,121.8,113.9,113.5,56.7$, 56.5, 39.9, 39.2, 32.5, 27.6; LC-MS: $m / z 495(\mathrm{M}+\mathrm{Na})^{+}$. Anal. Calcd for. $\mathrm{C}_{26} \mathrm{H}_{36} \mathrm{~N}_{2} \mathrm{O}_{6}, \mathrm{C}, 66.08 ; \mathrm{H}, 7.68 ; \mathrm{N}, 5.93$; Found: C, 66.03; H, 7.73; N, 5.98

$N, N^{\prime}$-(Butane-1,4-diyl)bis (3-(2,3,4,-trimethoxyphenyl) propanamide (4f). White solid, yield $90 \%, \mathrm{mp} 150-154^{\circ} \mathrm{C} .{ }^{1} \mathrm{H}$ NMR $\left(400 \mathrm{MHz}, \mathrm{DMSO}-d_{6}\right) \delta 7.82(\mathrm{t}, J=5.2 \mathrm{~Hz}, 2 \mathrm{H}), 6.89(\mathrm{~d}, J=8.4 \mathrm{~Hz}$, $2 \mathrm{H}), 6.76(\mathrm{~d}, J=8.8 \mathrm{~Hz}, 2 \mathrm{H}), 3.84(\mathrm{~s}, 6 \mathrm{H}), 3.82(\mathrm{~s}, 6 \mathrm{H}), 3.79(\mathrm{~s}, 6 \mathrm{H}), 3.07$ (d, $J=5.2 \mathrm{~Hz}, 4 \mathrm{H}), 2.77$ (t, $J=7.6 \mathrm{~Hz}, 4 \mathrm{H}), 2.34(\mathrm{t}, J=8 \mathrm{~Hz}, 4 \mathrm{H}), 1.39$ (brs, $4 \mathrm{H}) ;{ }^{13} \mathrm{C}-\mathrm{NMR}\left(100 \mathrm{MHz}, \mathrm{DMSO}-d_{6}\right) \delta 171.2,151.8,151.8,151.2$, 141.8, 126.8, 123.4, 107.8, 60.6, 60.6, 60.2, 55.8, 38.2, 36.3, 26.6, 25.1; LC-MS: $m / z 555(\mathrm{M}+\mathrm{Na})^{+}$. Anal. Calcd for. $\mathrm{C}_{28} \mathrm{H}_{40} \mathrm{~N}_{2} \mathrm{O}_{8}, \mathrm{C}, 63.14 ; \mathrm{H}$, 7.57; N, 5.26. Found: C,63.10; H, 7.62; N, 5.30.

$N, N^{\prime}$-(Butane-1,4-diyl)bis (3-(3,4,5-trimethoxyphenyl) propanamide $(\mathbf{4 g})$. White solid, yield $90 \%, \mathrm{mp} 140-144^{\circ} \mathrm{C} .{ }^{1} \mathrm{H}$ NMR $\left(400 \mathrm{MHz}, \mathrm{DMSO}-d_{6}\right) \delta 7.76(\mathrm{t}, J=5.2 \mathrm{~Hz}, 2 \mathrm{H}), 6.49(\mathrm{~s}, 4 \mathrm{H}), 3.74(\mathrm{~s}$, $6 \mathrm{H}), 3.62(\mathrm{~s}, 6 \mathrm{H}) 3.02(\mathrm{~d}, J=5.2 \mathrm{~Hz}, 4 \mathrm{H}), 2.75(\mathrm{t}, J=7.6 \mathrm{~Hz}, 4 \mathrm{H}), 2.35(\mathrm{t}$, $J=8.0 \mathrm{~Hz}, 4 \mathrm{H}), 1.33$ (brs, $2 \mathrm{H}) ;{ }^{13} \mathrm{C}-\mathrm{NMR}\left(100 \mathrm{MHz}, \mathrm{DMSO}-d_{6}\right) \delta 171.2$, 152.7, 137.1, 135.7, 105.5, 59.9, 55.7, 38.1, 37.1, 31.5, 26.6; LC-MS: m/z $533.4(\mathrm{M}+\mathrm{H})^{+}, 555.4(\mathrm{M}+\mathrm{Na})^{+}$. Anal. Calcd for. $\mathrm{C}_{28} \mathrm{H}_{40} \mathrm{~N}_{2} \mathrm{O}_{8}, \mathrm{C}, 63.14$; H, 7.57; N, 5.26. Found: C, 63.09; H, 7.64; N, 5.32.

$N, N^{\prime}$-(Butane-1,4-diyl)bis(3-(benzo [d] [ 1,3] dioxo]-5-yl) propanamide $(4 \mathbf{h})$. Brown solid, yield $88 \%, \mathrm{mp} 180-184^{\circ} \mathrm{C}$; ${ }^{1} \mathrm{H}$ NMR $\left(400 \mathrm{MHz}, \mathrm{DMSO}-d_{6}\right) \delta 7.75(\mathrm{t}, J=5.6 \mathrm{~Hz}, 2 \mathrm{H}), 6.78(\mathrm{~d}, J=8.0 \mathrm{~Hz}$, $2 \mathrm{H}), 6.76(\mathrm{~s}, 2 \mathrm{H}), 6.63(\mathrm{~d}, J=7.6 \mathrm{~Hz}, 2 \mathrm{H}), 5.94(\mathrm{~s}, 4 \mathrm{H}), 2.99(\mathrm{~m}, 4 \mathrm{H})$, $2.72(\mathrm{t}, J=7.6 \mathrm{~Hz}, 4 \mathrm{H}), 2.31(\mathrm{t}, J=7.6 \mathrm{~Hz}, 4 \mathrm{H}), 1.30$ (brs, $2 \mathrm{H}) .{ }^{13} \mathrm{C} \mathrm{NMR}$ (100 MHz, DMSO-d6): $\delta 171.1,147.1,145.2,135.2,120.9,108.6,107.9$, 100.5, 38.1, 37.3, 30.8, 26.5; LC-MS: $m / z$ 463.3 ( M+Na) ${ }^{+}$. Anal. Calcd for. $\mathrm{C}_{24} \mathrm{H}_{28} \mathrm{~N}_{2} \mathrm{O}_{6}, \mathrm{C}, 65.44 ; \mathrm{H}, 6.41 ; \mathrm{N}, 6.36$. Found: $\mathrm{C}, 65.39 ; \mathrm{H}, 6.45$; $\mathrm{N}, 6.40$.

$N, N^{\prime}$-(Butane-1,4-diyl)bis(3-(2-chlorophenyl)propanamide (4i). White solid, yield $86 \%$, mp162- $166^{\circ} \mathrm{C} .{ }^{1} \mathrm{H}$ NMR $\left(400 \mathrm{MHz}\right.$, DMSO- $\left.d_{6}\right)$ $\delta 7.81(\mathrm{~m}, 2 \mathrm{H}), 7.40(\mathrm{~d}, J=6.8 \mathrm{~Hz}, 2 \mathrm{H}), 7.28-7.14(\mathrm{~m}, 6 \mathrm{H}), 3.01$ (brs, $4 \mathrm{H}), 2.91(\mathrm{t}, J=8.0 \mathrm{~Hz}, 4 \mathrm{H}), 2.37(\mathrm{t}, J=7.6 \mathrm{~Hz}, 4 \mathrm{H}), 1.31$ (brs, $4 \mathrm{H}) .{ }^{13} \mathrm{C}$ NMR $\left(100 \mathrm{MHz}, \mathrm{DMSO}-d_{6}\right) \delta 171.0,141.4,130.4,129.1,128.2,127.2$, 125.8, 38.2, 37.0, 31.1, 26.6; LC-MS: $m / z 419.2(\mathrm{M}-\mathrm{H})$ - . Anal. Calcd for. $\mathrm{C}_{22} \mathrm{H}_{26} \mathrm{Cl}_{2} \mathrm{~N}_{2} \mathrm{O}_{2}, \mathrm{C}, 62.71 ; 6.22, \mathrm{Cl}, 16.83 ; \mathrm{N}, 6.65$ Found: C, 62.65; 6.28, $\mathrm{Cl}, 16.88 ; \mathrm{N}, 6.69$.

$N, N^{\prime}$-(Butane-1,4-diyl)dibenzamide (6a). White solid, yield 92\%, mp130-136 ${ }^{\circ} \mathrm{C} .{ }^{1} \mathrm{H}$ NMR (400 MHz, DMSO- $\left.d_{6}\right) \delta 8.49(\mathrm{t}, J=5.2 \mathrm{~Hz}$, $2 \mathrm{H}), 7.84(\mathrm{~d}, J=7.2 \mathrm{~Hz}, 4 \mathrm{H}), 7.51(\mathrm{t}, J=7.2 \mathrm{~Hz}, 2 \mathrm{H}), 7.44(\mathrm{t}, J=7.2$ $\mathrm{Hz}, 4 \mathrm{H}), 3.29$ (t, $J=6.4 \mathrm{~Hz}, 4 \mathrm{H}), 1.57$ (brs, $4 \mathrm{H}) .{ }^{13} \mathrm{C}$ NMR $(100 \mathrm{MHz}$,
DMSO- $\left.d_{6}\right) \delta 166.1,134.7,130.9,128.2,127.0,37.9,26.7$; LC-MS: $m / z$ 297.4 $(\mathrm{M}+\mathrm{H})^{+}, 329.4(\mathrm{M}+\mathrm{Na})^{+}$. Anal. Calcd for $\mathrm{C}_{18} \mathrm{H}_{20} \mathrm{~N}_{2} \mathrm{O}_{2}: \mathrm{C}, 72.95$, $\mathrm{H}, 6.80, \mathrm{~N}, 9.45$. Found C, 72.90, H, 6.85, N, 9.50.

$N, N^{\prime}$-(Butane-1,4-diyl)bis(4-methylbenzamide)

(6b). White solid, yield $92 \%$, mp $200-216^{\circ} \mathrm{C} .{ }^{1} \mathrm{H}$ NMR (400 MHz, DMSO- $d_{6}$ ) $\delta 8.39$ (t, $J=5.6 \mathrm{~Hz}, 2 \mathrm{H}), 7.74(\mathrm{~d}, J=8 \mathrm{~Hz}, 4 \mathrm{H}), 7.24(\mathrm{~d}, J=8 \mathrm{~Hz}, 4 \mathrm{H}), 3.27$ $(\mathrm{q}, J=5.6 \mathrm{~Hz}, 4 \mathrm{H}), 2.34(\mathrm{~s}, 6 \mathrm{H}), 1.55$ (brs, $4 \mathrm{H}) .{ }^{13} \mathrm{C}$ NMR $(100 \mathrm{MHz}$, DMSO- $\left.d_{6}\right) \delta 165.9,140.7,131.9,128.7,127.1,38.1,26.8,23.9$; LC-MS: $\mathrm{m} / z 325.4(\mathrm{M}+\mathrm{H})^{+}, 347.4(\mathrm{M}+\mathrm{Na})^{+}$. Anal. Calcd for. $\mathrm{C}_{20} \mathrm{H}_{24} \mathrm{~N}_{2} \mathrm{O}_{2}: \mathrm{C}$, 74.04; H, 7.46; N, 8.64. Found: C, 74.00; H, 7.50; N, 8.69.

$N, N^{\prime}$-(Butane-1,4-diyl)bis(3-methoxybenzamide) (6c). White solid, yield $92 \%$, mp $134-138^{\circ} \mathrm{C} .{ }^{1} \mathrm{H}$ NMR $\left(400 \mathrm{MHz}, \mathrm{DMSO}-d_{6}\right) \delta 8.39$ (brs, $2 \mathrm{H}), 7.37-7.28(\mathrm{~m}, 6 \mathrm{H}), 7.01(\mathrm{~d}, J=7.2 \mathrm{~Hz}, 2 \mathrm{H}), 3.73(\mathrm{~s}, 6 \mathrm{H}), 3.25$ $(\mathrm{t}, J=5.2 \mathrm{~Hz}, 4 \mathrm{H}), 1.51$ (brs, $2 \mathrm{H}) .{ }^{13} \mathrm{C}$ NMR $\left(100 \mathrm{MHz}\right.$, DMSO- $\left.d_{6}\right) \delta$ 165.8, 159.1, 136.1, 129.3, 119.3, 116.8, 112.4, 55.2, 38.9, 26.7; LC-MS: $\mathrm{m} / \mathrm{z} 379.4(\mathrm{M}+\mathrm{Na})^{+}$. Anal. Calcd for. $\mathrm{C}_{20} \mathrm{H}_{24} \mathrm{~N}_{2} \mathrm{O}_{4}, \mathrm{C}, 67.40 ; \mathrm{H}, 6.79 ; \mathrm{N}$, 7.86 Found: $\mathrm{C}, 67.35 ; \mathrm{H}, 6.83 ; \mathrm{N}, 7.90$.

$N, N^{\prime}$-(Butane-1,4-diyl)bis(3,5-dimethoxybenzamide) (6d). White solid, yield $90 \%, \mathrm{mp} 200^{\circ} \mathrm{C} .{ }^{1} \mathrm{H}$ NMR $\left(400 \mathrm{MHz}, \mathrm{DMSO}-d_{6}\right) \delta$ $8.47(\mathrm{t}, J=5.6 \mathrm{~Hz}, 2 \mathrm{H}) 7.01(\mathrm{~s}, J=2.0 \mathrm{~Hz}, 4 \mathrm{H}), 6.62(\mathrm{~s}, 2 \mathrm{H}), 3.77(\mathrm{~s}$, $12 \mathrm{H}), 3.27(\mathrm{t}, J=5.2 \mathrm{~Hz}, 4 \mathrm{H}), 1.55$ ( brs, $4 \mathrm{H}) .{ }^{13} \mathrm{C}$ NMR $(100 \mathrm{MHz}$, DMSO-d $d_{6} \delta 165.6,160.3,136.8,105.1,102.9,55.3,38.0,26.6$, LC-MS: $m / z 417(\mathrm{M}+\mathrm{H})^{+}$. Anal. Calcd for. $\mathrm{C}_{22} \mathrm{H}_{28} \mathrm{~N}_{2} \mathrm{O}_{6}: \mathrm{C}, 63.45 ; \mathrm{H}, 6.78 ; \mathrm{N}$, 6.73. Found C, 63.40; H, 6.82; N, 6.78 .

$N, N^{\prime}$-(Butane-1,4-diyl)bis(4-fluorobenzamide) (6e). White solid, yield $85 \%$, mp $224-230^{\circ} \mathrm{C}^{1} \mathrm{H}$ NMR (400 MHz, DMSO- $\left.d_{6}\right) \delta 8.56(\mathrm{t}, J=$ $5.6 \mathrm{~Hz}, 2 \mathrm{H}), 7.93(\mathrm{dd}, J=5.6,8.8 \mathrm{~Hz}, 4 \mathrm{H}), 7.27(\mathrm{t}, J=8.8 \mathrm{~Hz}, 4 \mathrm{H}), 3.28$ $(\mathrm{t}, J=5.2 \mathrm{~Hz}, 4 \mathrm{H}), 1.56$ (brs, $4 \mathrm{H}) .{ }^{13} \mathrm{C}-\mathrm{NMR}\left(100 \mathrm{MHz}, \mathrm{DMSO}-d_{6}\right) \delta$ 165.0, 161.3, 131.1, 129.7, 115.1, 38.0, 26.6; LC-MS: $m / z 331$ (M-H) . Anal. Calcd for. $\mathrm{C}_{18} \mathrm{H}_{18} \mathrm{~F}_{2} \mathrm{~N}_{2} \mathrm{O}_{2}: \mathrm{C}, 65.05 ; \mathrm{H}, 5.46 ; \mathrm{F}, 11.43 ; \mathrm{N}, 8.43$, Found: C, 65.00; H, 5.51; F, 11.48; N, 8.47.

$N, N^{\prime}$-(Butane-1,4-diyl)bis(2-iodobenzamide) (6f). White solid, yield $90 \%, \mathrm{mp} 210-218^{\circ} \mathrm{C}^{1} \mathrm{H}$ NMR $\left(400 \mathrm{MHz}, \mathrm{DMSO}-d_{6}\right) \delta 8.36(\mathrm{t}, J=$ $5.2 \mathrm{~Hz}, 2 \mathrm{H}), 7.87(\mathrm{~d}, J=7.6 \mathrm{~Hz}, 2 \mathrm{H}), 7.43(\mathrm{t}, J=7.6 \mathrm{~Hz}, 2 \mathrm{H}), 7.31(\mathrm{dd}, J$ $=1.2,7.6 \mathrm{~Hz}, 2 \mathrm{H}), 7.15(\mathrm{td}, J=1.6,8.0 \mathrm{~Hz}, 2 \mathrm{H}), 3.25(\mathrm{t}, J=5.2 \mathrm{~Hz}, 4 \mathrm{H})$, $1.63(\mathrm{~m}, 4 \mathrm{H}) .{ }^{13} \mathrm{C}-\mathrm{NMR}\left(100 \mathrm{MHz}, \mathrm{DMSO}-d_{6}\right) \delta 168.7,143.4,138.9$, $130.5,127.9,127.8,93.4,38.0,26.4$; LC-MS: $m / z 549(\mathrm{M}+\mathrm{H})^{+}, 571($ $\mathrm{M}+\mathrm{Na})^{+}$. Anal. Calcd for. $\mathrm{C}_{18} \mathrm{H}_{18} \mathrm{I}_{2} \mathrm{~N}_{2} \mathrm{O}_{2}: \mathrm{C}, 39.44 ; \mathrm{H}, 3.31 ; \mathrm{I}, 46.30 ; \mathrm{N}$, 5.11, Found: C, 39.40; H, 3.35; I, 46.34; N, 5.16

$N, N^{\prime}$-(Butane-1,4-diyl)bis(2,4-dichlorobenzamide) (6g). White solid, yield 90\%, mp $232-238^{\circ} \mathrm{C},{ }^{1} \mathrm{H}$ NMR (400 MHz, DMSO- $d_{6}$ ) $\delta 8.49$ $(\mathrm{t}, J=5.6 \mathrm{~Hz}, 2 \mathrm{H}), 7.67(\mathrm{~d}, J=1.6 \mathrm{~Hz}, 2 \mathrm{H}), 7.48(\mathrm{~d}, J=2.0,8.0 \mathrm{~Hz}, 2 \mathrm{H})$, $7.45(\mathrm{~d}, J=8.4 \mathrm{~Hz}, 2 \mathrm{H}), 3.25(4 \mathrm{H}, \mathrm{t}, J=5.2 \mathrm{~Hz}, 4 \mathrm{H}), 1.57(\mathrm{~m}, 4 \mathrm{H}) .{ }^{13} \mathrm{C}$ NMR (100 MHz, DMSO- $\left.d_{6}\right) \delta 165.2,136.1,134.2,131.1,130.1,129.0$, 127.2, 38.0, 26.3; LC-MS: $m / z 435.5(\mathrm{M}+\mathrm{H})^{+}, 457(\mathrm{M}+\mathrm{Na})^{+}$Anal. Calcd for. $\mathrm{C}_{18} \mathrm{H}_{16} \mathrm{C}_{14} \mathrm{~N}_{2} \mathrm{O}_{2}: \mathrm{C}, 49.80 ; \mathrm{H}, 3.71 ; \mathrm{Cl}, 32.66$; N. 6.45, Found: C, $49.75 ; \mathrm{H}, 3.75 ; \mathrm{Cl}, 38.66$; N. 6.49 .

$\boldsymbol{N}, \boldsymbol{N}^{\prime}$-(Butane-1,4-diyl)diacetamide (6h). Pale yellow solid, yield 90\%, mp $120-128^{\circ} \mathrm{C} .{ }^{1} \mathrm{H}$ NMR (400 MHz, DMSO- $\left.d_{6}\right) \delta 7.82(\mathrm{~s}, 2 \mathrm{H}), 3.00$ $(\mathrm{s}, 4 \mathrm{H}), 1.77$ (s, 6H, 1.35 (s, 4H). ${ }^{13} \mathrm{C}-\mathrm{NMR}\left(100 \mathrm{MHz}\right.$, DMSO- $\left.d_{6}\right) 168.8$, 38.1, 26.5, 22.4; LC-MS: $m / z 195(\mathrm{M}+\mathrm{Na})^{+}$Anal. Calcd for. $\mathrm{C}_{8} \mathrm{H}_{16} \mathrm{~N}_{2} \mathrm{O}_{2}$, C, 55.79; H, 9.36; N, 16.27, Found: C, 55.74; H, 9.39; N, 16.32 .

$N, N^{\prime}$-(Butane-1,4-diyl)dipropionamide (6i). Pale yellow solid, yield $90 \%, \mathrm{mp} 120^{\circ} \mathrm{C}{ }^{1} \mathrm{H}$ NMR $\left(400 \mathrm{MHz}\right.$, DMSO- $\left.d_{6}\right) \delta 7.74(\mathrm{~s}, 2 \mathrm{H}) 3.01$ $(\mathrm{q}, J=5.2 \mathrm{~Hz}, 4 \mathrm{H}), 2.05(\mathrm{q}, J=7.2 \mathrm{~Hz}, 4 \mathrm{H}), 1.36(\mathrm{~m}, 4 \mathrm{H}), 0.97$ (t, $J=7.6$ $\mathrm{Hz}, 6 \mathrm{H}) .{ }^{13} \mathrm{C}-\mathrm{NMR}\left(100 \mathrm{MHz}, \mathrm{DMSO}-d_{6}\right) \delta 172.7,38.5,28.6,26.6,9.9$. $m / z 223(\mathrm{M}+\mathrm{Na})^{+}$. Anal. Calcd for. $\mathrm{C}_{10} \mathrm{H}_{20} \mathrm{~N}_{2} \mathrm{O}_{2}, \mathrm{C}, 59.97 ; \mathrm{H}, 10.07 ; \mathrm{N}$, 
13.99, Found: C, 59.92; H, 10.12; N, 14.04 .

$N, N^{\prime}$-(Butane-1,4-diyl)dihexanamide (6j). Pale yellow solid, mp154-160 ${ }^{\circ} \mathrm{C} .{ }^{1} \mathrm{H}$ NMR $\left(400 \mathrm{MHz}, \mathrm{DMSO}-d_{6}\right) \delta 7.77$ (brs, $\left.2 \mathrm{H}\right), 3.06$ $(\mathrm{q}, J=5.2 \mathrm{~Hz}, 4 \mathrm{H}), 2.08(\mathrm{t}, J=7.2 \mathrm{~Hz}, 4 \mathrm{H}), 1.53(\mathrm{~m}, 4 \mathrm{H}), 1.41$ (brs, $4 \mathrm{H}), 1.33-1.24(\mathrm{~m}, 8 \mathrm{H}), 0.91(\mathrm{t}, J=7.2 \mathrm{~Hz}, 6 \mathrm{H}) .{ }^{13} \mathrm{C}-\mathrm{NMR}(100 \mathrm{MHz}$, DMSO- $\left.d_{6}\right) \delta 171.9,38.1,35.4,30.9,26.7,24.9,21.8,13.8 ;$ LC-MS: $m / z$ $307(\mathrm{M}+\mathrm{Na})^{+}$. Anal. Calcd for. $\mathrm{C}_{16} \mathrm{H}_{32} \mathrm{~N}_{2} \mathrm{O}_{2}, \mathrm{C}, 67.56 ; \mathrm{H}, 11.34 ; \mathrm{N}, 9.85$, Found: C, 67.50; H, 11.40; N, 9.89.

\section{Results and Discussion}

In this study, we have synthesized JBIR-94 along with nine structural analogs and a series of substituted phenyl and dialkyl putrescine bisamides for the first time. We have also screened these compounds for antimicrobial, 5-lipoxynase enzyme inhibitory and antioxidant activities.

\section{Chemistry}

Benzyl protected ferulic acid was coupled with putrescine via acid chloride which leads to the formation of $N_{1}, N_{6}$-diferulic putrescine bisamide. Subsequently it was reduced and deprotected with $\mathrm{Pd} / \mathrm{CaCO}_{3}$ to afford the desired product JBIR-94 in good yields (Scheme 1). All the spectral data of the synthesized compound is in good agreement after with isolated compound. The other structural analogues $4 \mathrm{~b}-4 \mathrm{i}$ have been synthesized from putrescine and appropriately substituted cinnamic acids as shown in Scheme 1.

Similarly, we have synthesized a series of $N_{1}, N_{6}$-substituted phenyl and $N_{1}, N_{6}$-alkyl putrescine bisamides with putrescine by varying aromatic/aliphatic acid chlorides Scheme 2. All the compounds were well characterized by advanced spectroscopic techniques like ${ }^{1} \mathrm{H} N M R$, ${ }^{13} \mathrm{C}$ NMR and Mass (Tables 1 and 2).

\section{Bioactivity}

Antimicrobial activity: Antimicrobial studies were carried out on clinical isolates of human pathogenic bacteria (Salmonella typhi, Vibrio cholerae, Shigella dysenteriae, Enterococcus faecalis and Staphylococcus aureus) and dermatophytic fungi. (Candida albicans). As shown in Table 3, newly synthesized putrescine bisamides showed significant antibacterial activity but poor anti-fungal activity. Compounds inhibitory zones were found to be in the range between $2-14 \mathrm{~mm}$ while $1-1000 \mu \mathrm{g} / \mathrm{ml}$ was the MIC range of compounds. In $N_{1}, N_{6}$ dihydrocinnamyl putrescine bisamide series (4a- 4i), 4h, 4a (JBIR-94) and $4 \mathrm{f}$ showed potent antimicrobial activity but compounds, $4 \mathrm{c}, 4 \mathrm{~b}$, $4 \mathrm{~d}, 4 \mathrm{e}, 4 \mathrm{~g}$, and $4 \mathrm{i}$ showed moderate activity. Substituted phenyl (6a$6 \mathrm{~g}$ ) and dialkyl (6h-6j) series of putrescine bisamides showed poor antimicrobial activity. Among all the tested compounds, $4 \mathrm{~h}$ showed highest $(14 \mathrm{~mm})$ inhibitory zone and lowest MIC value $(1 \mu \mathrm{g} / \mathrm{ml})$ on S. typhi. Moreover it showed comparable potency with Ciprofloxacin<smiles>COc1cc(CCC(=O)NCCCCNC(=O)CCc2ccc(O)c(OC)c2)ccc1O</smiles>

JBIR-94

\begin{tabular}{|c|c|c|c|c|c|c|c|}
\hline S.No & Entry & $\mathbf{R}_{\mathbf{1}}$ & $\mathbf{R}_{\mathbf{2}}$ & $\mathbf{R}_{\mathbf{3}}$ & $\mathbf{R}_{\mathbf{4}}$ & $\mathbf{R}_{\mathbf{5}}$ & $\mathbf{4 Y \text { Yield(\%) }}$ \\
\hline 1 & $\mathbf{4 a}$ (JBIR) & $\mathrm{H}$ & $\mathrm{OMe}$ & $\mathrm{OH}$ & $\mathrm{H}$ & $\mathrm{H}$ & 88 \\
\hline 2 & $4 \mathrm{~b}$ & $\mathrm{H}$ & $\mathrm{OMe}$ & $\mathrm{H}$ & $\mathrm{H}$ & $\mathrm{H}$ & 90 \\
\hline 3 & $4 \mathrm{c}$ & $\mathrm{OMe}$ & $\mathrm{H}$ & $\mathrm{H}$ & $\mathrm{H}$ & $\mathrm{H}$ & 90 \\
\hline 4 & $4 \mathrm{~d}$ & $\mathrm{H}$ & $\mathrm{H}$ & $\mathrm{OMe}$ & $\mathrm{H}$ & $\mathrm{H}$ & 90 \\
\hline 5 & $4 \mathrm{e}$ & $\mathrm{H}$ & $\mathrm{OMe}$ & $\mathrm{OMe}$ & $\mathrm{H}$ & $\mathrm{H}$ & 89 \\
\hline 6 & $4 \mathrm{f}$ & $\mathrm{OMe}$ & $\mathrm{OMe}$ & $\mathrm{OMe}$ & $\mathrm{H}$ & $\mathrm{H}$ & 88 \\
\hline 7 & $4 \mathrm{~g}$ & $\mathrm{H}$ & $\mathrm{OMe}$ & $\mathrm{OMe}^{\mathbf{O}}$ & $\mathrm{OMe}$ & $\mathrm{H}$ & 87 \\
\hline 8 & $4 \mathrm{~h}$ & $\mathrm{H}$ & & $\mathrm{oCH}_{2} \mathrm{O}$ & $\mathrm{H}$ & $\mathrm{H}$ & 86 \\
\hline 9 & $4 \mathrm{i}$ & $\mathrm{Cl}$ & $\mathrm{H}$ & $\mathrm{H}$ & $\mathrm{H}$ & $\mathrm{H}$ & 85 \\
\hline
\end{tabular}

alsolated yields

Table 1: Synthesis of $N_{1}, N_{6}$-dihydrocinnamyl putrescine bisamides.

\begin{tabular}{|c|c|c|c|}
\hline S. No & Entry & $\mathbf{R}$ & $\mathbf{6 Y i e l d ~ ( \% ) ~}^{\mathbf{a}}$ \\
\hline 1 & $6 \mathrm{a}$ & $\mathrm{Ph}$ & 92 \\
\hline 2 & $6 \mathrm{~b}$ & $4-\mathrm{Me} \mathrm{Ph}$ & 92 \\
\hline 3 & $6 \mathrm{c}$ & 3-OMe Ph & 90 \\
\hline 4 & $6 \mathrm{~d}$ & 3,4-diOMe Ph & 90 \\
\hline 5 & $6 \mathrm{e}$ & $4-\mathrm{F} \mathrm{Ph}$ & 86 \\
\hline 6 & $6 f$ & $2-\mathrm{IPh}$ & 88 \\
\hline 7 & $6 \mathrm{~g}$ & $2,4-\mathrm{diCl} \mathrm{Ph}$ & 86 \\
\hline 8 & $6 \mathrm{M}$ & $\mathrm{Me}$ & 94 \\
\hline 9 & $6 \mathrm{i}$ & $\mathrm{Et}$ & 94 \\
\hline 10 & $6 j$ & Pentyl & 92 \\
\hline
\end{tabular}

alsolated yields

Table 2: Synthesis of $N_{1}, N_{6}$-phenyl and $N_{1}, N_{6}$-alkyl putrescine bisamides.

\begin{tabular}{|c|c|c|c|c|c|c|}
\hline \multirow[b]{2}{*}{ Entry $^{+}$} & \multicolumn{4}{|c|}{ Zone of inhibition $(\mathrm{mm}) / \mathrm{MIC}(\mu \mathrm{g} / \mathrm{ml})$} & \multirow[b]{2}{*}{$\begin{array}{l}\text { S. } \\
\text { aureus }\end{array}$} & \multirow[b]{2}{*}{$\begin{array}{l}\text { C. } \\
\text { albicans }\end{array}$} \\
\hline & $\begin{array}{l}\text { S. } \\
\text { typhi }\end{array}$ & $\begin{array}{l}\text { V. } \\
\text { cholerae }\end{array}$ & $\begin{array}{l}\text { S. } \\
\text { dysenteriae }\end{array}$ & $\begin{array}{l}\text { E. } \\
\text { faecalis }\end{array}$ & & \\
\hline $4 a$ & $8 / 100$ & $8 / 10$ & $7 / 100$ & $8 / 100$ & $9 / 100$ & $7 / 100$ \\
\hline $4 b$ & $4 / 100$ & $5 / 100$ & $2 / 100$ & ND & $6 / 100$ & $6 / 100$ \\
\hline $4 c$ & $5 / 1000$ & $4 / 100$ & $3 / 100$ & $5 / 100$ & $4 / 100$ & ND \\
\hline $4 d$ & $4 / 100$ & $5 / 100$ & $4 / 100$ & $3 / 1000$ & $4 / 100$ & $2 / 100$ \\
\hline $4 e$ & $5 / 100$ & $6 / 100$ & $5 / 100$ & $5 / 100$ & $6 / 1000$ & $4 / 100$ \\
\hline $4 f$ & $7 / 100$ & $8 / 1000$ & $7 / 100$ & $8 / 100$ & $7 / 1000$ & $6 / 1000$ \\
\hline $4 \mathrm{~g}$ & $5 / 1000$ & $5 / 100$ & $6 / 100$ & $7 / 1000$ & $8 / 100$ & $7 / 100$ \\
\hline $4 \mathrm{~h}$ & $14 / 1$ & $13 / 10$ & $12 / 10$ & $12 / 10$ & $13 / 10$ & $10 / 100$ \\
\hline $4 i$ & $6 / 10$ & $7 / 100$ & $7 / 1000$ & $7 / 100$ & $6 / 100$ & $5 / 100$ \\
\hline $6 a$ & $5 / 100$ & $5 / 100$ & $6 / 1000$ & $5 / 1000$ & $7 / 100$ & $5 / 100$ \\
\hline $6 b$ & $4 / 100$ & $5 / 100$ & $5 / 100$ & $4 / 10$ & $5 / 100$ & $4 / 100$ \\
\hline $6 c$ & $5 / 1000$ & $4 / 1000$ & $6 / 100$ & $5 / 1000$ & $4 / 1000$ & $5 / 100$ \\
\hline $6 d$ & $5 / 1000$ & $4 / 1000$ & $6 / 100$ & $7 / 100$ & $5 / 1000$ & $4 / 1000$ \\
\hline $6 e$ & $4 / 1000$ & $5 / 1000$ & $5 / 1000$ & $4 / 1000$ & $5 / 100$ & $4 / 1000$ \\
\hline $6 f$ & $3 / 1000$ & $4 / 1000$ & $3 / 100$ & $3 / 100$ & $4 / 100$ & $2 / 1000$ \\
\hline $6 \mathrm{~g}$ & $2 / 1000$ & $2 / 100$ & $3 / 100$ & $3 / 1000$ & $4 / 1000$ & $2 />1000$ \\
\hline $6 \mathrm{~h}$ & $2 / 1000$ & $2 / 1000$ & $4 / 10$ & $3 / 100$ & $4 / 1000$ & $2 / 1000$ \\
\hline $6 i$ & $3 / 1000$ & $4 / 100$ & $3 / 1000$ & $2 / 100$ & $4 / 1000$ & ND \\
\hline $6 \mathrm{j}$ & $4 / 1000$ & $3 / 1000$ & $5 / 100$ & ND & $4 / 1000$ & ND \\
\hline Standard* & $12 / 10$ & $12 / 1$ & $13 / 10$ & $12 / 10$ & $11 / 10$ & $11 / 10$ \\
\hline
\end{tabular}

*Ciprofloxacin for bacteria and Griseofulvin for dermatophytic fungi; ${ }^{+} 50 \mu \mathrm{g}$ compound per well; ND: Not Determined

Table 3: Antimicrobial activities of newly synthesized putrescine bisamide derivatives against various human pathogens.

which is an antibiotic used as positive control.

5-Lipoxygenase inhibitory activity: All synthesized putrescine bisamides were evaluated for 5-Lipoxygenase assay and found to have significant 5-LOX inhibitory activity with $\mathrm{IC}_{50}$ range from 9.2 to 29.2 $\mu \mathrm{g} / \mathrm{ml}$ (Table 4). Dihydrocinnamyl series of compounds (4a-4i), and substituted phenyl (6a-6g) series of compounds showed effective enzyme inhibitory activity with $\mathrm{IC}_{50}$ range from 9.2 to $14.6 \mu \mathrm{g} / \mathrm{ml}$, 
Citation: Rayavarapu S, Kadiri SK, Gajula MB, Nakka M, Tadikonda R, et al. (2014) Synthesis of Putrescine Bisamides as Antimicrobial and AntiInflammatory Agents. Med chem 4: 367-372. doi:10.4172/2161-0444.1000167

dialkyl series of compounds $6 \mathrm{~h}, 6 \mathrm{i}$ and $6 \mathrm{j}$ showed dose dependent inhibition with an $\mathrm{IC}_{50}$ of $21,26.5$ and $29.2 \mu \mathrm{g} / \mathrm{ml}$ respectively. These results demonstrated that with the increase in aliphatic carbon chain in dialkyl series causes the decreasing inhibitory activity. Among all the tested compounds $4 \mathrm{a}$ (JBIR-94) and $4 \mathrm{~h}$ (methylenedioxy analog) showed potent inhibitory activity with $\mathrm{IC}_{50}$ of 9.7 and $9.2 \mu \mathrm{g} / \mathrm{ml}$ respectively. Nordihydroguaiaretic acid (positive control) inhibited 5-LOX with $\mathrm{IC}_{50}$ of $4.40 \mu \mathrm{g} / \mathrm{ml}$.

Further molecular docking studies of all synthesized putrescine bisamides were performed on 5-Lipoxygenase crystal protein (PDB ID 308Y) using iGEM dock programme and found the docking scores (binding energies) range between -98.92 - $-141.23 \mathrm{Kcal} / \mathrm{mol}$ (Table 4). Among all compounds, $4 \mathrm{a}$ and $4 \mathrm{~h}$ showed good docking efficiency, which was comparable with Nordihydroguaiaretic acid (Positive control). Docking of 5-LOX with 4a (JBIR-94) showed the binding energy of $-138.67(\mathrm{kcal} / \mathrm{mol})$ and binds the vicinity of amino acid residues present at active site were $\mathrm{Leu}^{188}, \mathrm{Ala}^{561}, \mathrm{Asn}^{187}$ and Ser ${ }^{562} .4 \mathrm{~h}$ binds the vicinity of $\mathrm{Leu}^{188}$, Asp ${ }^{368}$, Ser ${ }^{562}$, Asn187 amino acid residues of 5-LOX crystal protein with the binding energy of $-141.23 \mathrm{kcal} / \mathrm{mol}$ (Figure 1).

Among all synthesized putrescine bisamides, $4 \mathrm{a}$ and $4 \mathrm{~h}$ showed good in vitro 5-LOX inhibition activities and also possess good binding capability with catalytic amino acids of 5-LOX in molecular docking studies (Figure 1).

\section{Antioxidant activity}<smiles>[R]c1c([R])c([R])c(/C=C/C(=O)O)c([R])c1[R]</smiles>

1<smiles>[R]Cc1c([R])c([R])c(/C=C/C(=O)NCCCCNC(=O)/C=C/c2c([R])c([R])c([R])c([R])c2[R])c([R])c1[R]</smiles>

3<smiles>[R]Cc1c([R])c([R])c([R])c(CCC(=O)NCCCCNC(=O)CCc2c([R])c([R])c([R3])c([R])c2[R])c1[R]</smiles>

4

Reagents and condition: (a) $\mathrm{SOCl}_{2}$, Putrescine (2), TEA, 0.5 h, rt; (b) $\mathrm{Pd} / \mathrm{CaCO}_{3}$, rt, 2 h.<smiles>[R]C(=O)NCCCCNC([R])=O</smiles>

Reagents and conditions: (c) Putrescine, TEA, DCM, $0.5 \mathrm{~h}$

Scheme 2

\begin{tabular}{|c|c|c|c|c|}
\hline \multirow{2}{*}{ Entry } & \multicolumn{2}{|c|}{ 5-LOX inhibitory activity (Anti-inflammatory activity) } & \multicolumn{2}{|c|}{ Antioxidant activity (Radical scavenging activity) } \\
\hline & In vitro studies $\mathrm{IC}_{50}(\mu \mathrm{g} / \mathrm{ml})$ & Insilico studies Dock score (Kcal/mol) & DPPH & Superoxide $\mathrm{IC}_{50}(\mu \mathrm{g} / \mathrm{ml})$ \\
\hline $4 a$ & 9.7 & -138.67 & 10.99 & 9.8 \\
\hline $4 b$ & ND & -110.76 & 8.3 & 7.8 \\
\hline $4 c$ & 13.1 & -129.32 & 8.5 & 7.6 \\
\hline $4 d$ & 13.3 & -128.21 & 8.8 & 7.5 \\
\hline $4 e$ & 14.1 & -124.87 & 10.0 & 9.1 \\
\hline $4 f$ & 15.4 & -119.67 & 9.5 & 9.8 \\
\hline $4 g$ & 15.3 & -118.56 & 15.4 & 19.8 \\
\hline $4 \mathrm{~h}$ & 9.2 & -141.23 & 5.6 & 7.6 \\
\hline $4 i$ & 12.2 & -122.65 & 13.1 & 14.2 \\
\hline $6 a$ & 14.6 & -126.61 & 14.2 & 14.3 \\
\hline $6 b$ & 13.4 & -121.01 & 10.0 & 13.1 \\
\hline $6 c$ & 13.5 & -127.21 & 9.3 & 10.3 \\
\hline $6 d$ & 14.4 & -129.34 & 12.2 & 10.9 \\
\hline $6 e$ & 10.4 & -118.32 & 15.5 & 13.03 \\
\hline $6 f$ & 10.1 & -108.49 & 16.43 & 15.02 \\
\hline $6 g$ & 10.1 & -119.94 & 14.45 & 13.5 \\
\hline $6 \mathrm{~h}$ & 21.0 & -102.56 & 8.7 & 10.3 \\
\hline $6 i$ & 26.5 & -99.23 & 10.2 & 11.2 \\
\hline $6 j$ & 29.2 & -98.92 & 11.2 & 11.9 \\
\hline Standard* & 4.40 & -154.92 & 3.6 & 3.3 \\
\hline
\end{tabular}

*Nordihydroguaiaretic acid for 5-LOX, Ascorbic acid for antioxidant activity, ND; Not Determined

Table 4: Anti-inflammatory and antioxidant activities of newly synthesized putrescine bisamides. 


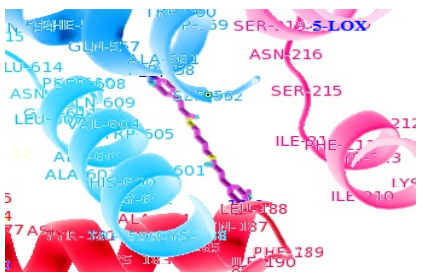

(A). 4a (JBIR-94) binds vicinity of $\mathrm{Lu}^{188}$ Ala ${ }^{561}$ Asn ${ }^{187}$, Ser 562 amino acid residues of 5-LOX crystal protein with binding energy of $-138.67 \mathrm{kcal} / \mathrm{mol}$.

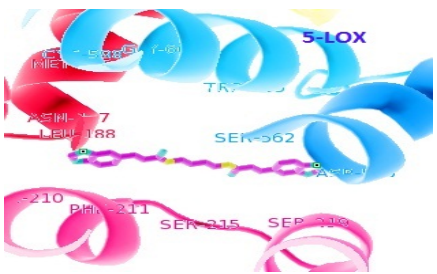

(B). $4 \mathrm{~h}$ binds in the vicinity of Leu ${ }^{188}$, Asp $^{368}$, Ser ${ }^{562}, \quad$ Asn $^{187}$ amino acid residues of 5 -LOX crystal protein with binding energy of $-141.23 \mathrm{kcal} / \mathrm{mol}$.
Figure 1: Molecular docking studies and interaction analysis of $4 \mathrm{a}$ and $4 \mathrm{~h}$ on 5-Lipoxygenase enzyme crystal sructure (PDB ID 308Y).

Antioxidant activities of putrescine bisamides were performed through radical scavenging assay using DPPH and Superoxide radicals. Dihydrocinnamyl (4a-4i), substituted phenyl (6a-6g) and dialkyl (6h6j) series of putrescine bisamides showed good free radical scavenging activity. Among all the tested compounds, $4 \mathrm{~h}$ (methylenedioxy analog) was finest radical scavenger with an $\mathrm{IC}_{50}$ of 5.6 and $7.6 \mu \mathrm{g} / \mathrm{ml}$ for DPPH and Superoxide radicals respectively.

In conclusion, a new naturally occuring $N_{1}, N_{6}$-didihydrocinnamyl putrescine bisamide, JBIR-94, along with nine structural analogs and a series of substituted phenyl and alkyl putrescine bisamides have been synthesized from putrescine and appropriately substituted carboxylic acids. Antimicrobial, 5-LOX enzyme inhibitory and antioxidant studies were performed for all synthesized compounds. Dihydrocinnamyl series of putrescine bisamides (4a-4i) showed efficient bioactivities compared to substituted phenyl (6a-6g) and dialkyl (6h-6j) series of compounds. Among the tested compounds, $4 \mathrm{~h}$ and $4 \mathrm{a}$ (JBIR94) showed good antimicrobial, anti-inflammatory and antioxidant activities.

\section{Acknowledgement}

The authors thank to the University Grants Commission (UGC/MRP F.No37$1 / 2009$ (AP)(SR), New Delhi for financial assistance (Through a project No 37 $1 / 2009$ (AP)(SR). The authors are thankful to Laila Impex R\&D center, Vijayawada for providing spectral data and laboratory facilities.

\section{References}

1. Richard D, Manfred H (2002) Synthesis and structure elucidation of openchained putrescine-bisamides from Aglaiaspecies. Tetrahedron. 58: 68876893.

2. Harald G, Thomas P, Brigitte B, Markus B, Otmar H (2001) Insecticidal flavaglines and other compounds from Fijian Aglaila species. Phytochem 57: 57-64

3. Nugrohoa BW, Edradaa RA, Wrayb V, Wittec L, Bringmannd G, et al. (1999) An insecticidal rocaglamide derivatives and related compounds from Aglaia odorata (Meliaceae). Phyto chem 51:367-376.

4. Abdelfattah MS, Toume K, Ahmed F, Sadhu SK, Ishibashi M (2010) Cucullamide, a new putrescine bisamide from Amoora cucullata. Chem Pharm Bull (Tokyo) 58: 1116-1118.

5. Dehaghi N, Lai D, Amanzadeh Y, Ebrahimi S, Proksch P. (2012) The effect of high partial pressures of oxygen on photosynthesis in Chlorella-l. Phyto chem Lett. 5: 643-646.

6. Kim EO, Min KJ, Kwon TK, Um BH, Moreau RA, et al. (2012) Anti-inflammatory activity of hydroxycinnamic acid derivatives isolated from corn bran in lipopolysaccharide-stimulated Raw 264.7 macrophages. Food Chem Toxicol 50: $1309-1316$

7. Kim K, Choi S, Lee K. (2012) Turtschamide, a cytotoxic putrescinebisamide from Corydalis turtschaninovii. Tetrahedron Lett 53:1490-1492.

8. Niwa T, Doi U, Osawa T (2003) Inhibitory activity of corn-derived bisamide compounds against alpha-glucosidase. J Agric Food Chem 51: 90-94.

9. Kawahara $T$, Izumikawa $M$, Otoguro $M$, Yamamura $H$, Hayakawa $M$, et al. (2012) JBIR-94 and JBIR-125, antioxidative phenolic compounds from Streptomyces sp. R56-07. J Nat Prod 75: 107-110.

10. Choi SW, Lee SK, Kim EO, Oh JH, Yoon KS, et al. (2007) Antioxidant and antimelanogenic activities of polyamine conjugates from corn bran and related hydroxycinnamic acids. J Agric Food Chem 55: 3920-3925

11. Brader G, Vajrodaya S, Greger H, Bacher M, Kalchhauser H, et al. (1998) Bisamides, lignans, triterpenes, and insecticidal Cyclopenta[b]benzofurans from Aglaia species. J Nat Prod 61: 1482-1490.

12. Mellon JE, Moreau RA (2004) Inhibition of aflatoxin biosynthesis in Aspergillus flavus by diferuloylputrescine and p-coumaroylferuloylputrescine. J Agric Food Chem 52: 6660-6663.

13. Duong TN, Edrada R, Ebel R, Wray V, Frank W, et al. (2007) Putrescine bisamides from Aglaia gigantea. J Nat Prod 70: 1640-1643.

14. Dobrikov GM, Valcheva V, Stoilova-Disheva M, Momekov G, Tzvetkova P, et al (2012) Synthesis and inÂ vitro antimycobacterial activity of compounds derived from $(R)$ - and $(S)$-2-amino-1-butanol - The crucial role of the configuration. Eur J Med Chem 48: 45-56.

15. Pfaller MA, Castanheira M, Diekema DJ, Messer SA, Jones RN (2011) Triazole and echinocandin MIC distributions with epidemiological cutoff values for differentiation of wild-type strains from non-wild-type strains of six uncommon species of Candida. J Clin Microbiol 49: 3800-3804.

16. Aparoy P, Reddy RN, Guruprasad L, Reddy MR, Reddanna P (2008) Homology modeling of 5-lipoxygenase and hints for better inhibitor design. J Comput Aided Mol Des 22: 611-619.

17. Reddy NP, Aparoy P, Reddy TC, Achari C, Sridhar PR, et al. (2010) Design, synthesis, and biological evaluation of prenylated chalcones as 5-LOX inhibitors. Bioorg Med Chem 18: 5807-5815.

18. Reddanna P, Whelan J, Maddipati KR, Reddy CC (1990) Purification of arachidonate 5-lipoxygenase from potato tubers. Methods Enzymol 187: 268277

19. Lamaison JL, Petitjean-Freytet C, Carnat A (1991) [Medicinal Lamiaceae with antioxidant properties, a potential source of rosmarinic acid]. Pharm Acta Helv 66: 185-188.

20. McCord JM, Fridovich I (1969) Superoxide dismutase. An enzymic function for erythrocuprein (hemocuprein). J Biol Chem 244: 6049-6055.

21. Ruby JA, Grigakutlan JK, Babu KVD, sekarau KNR, Kuttan R (1996) Antitumour and free radical scavenging activity of synthetic curcuminoids. Int $J$ Pharm 131:1-7.

22. Yang JM (2004) Development and evaluation of a generic evolutionary method for protein-ligand docking. J Comput Chem 25: 843-857.

23. Yang JM, Chen CC (2004) GEMDOCK: a generic evolutionary method for molecular docking. Proteins 55: 288-304.

24. Lua RC, Lichtarge O (2010) PyETV: a PyMOL evolutionary trace viewer to analyze functional site predictions in protein complexes. Bioinformatics 26 : 2981-2982.

25. Eswar kumar K, Swathi P, Nagendra sastry Y, Kaladhar DSVGK, Govinda rao D (2012) M07-A9 Methods for Dilution Antimicrobial Susceptibility Test for Bacteria That Grow Aerobically; Approved Standard - Ninth Edition 32: 12-20.

26. Usharani A, Bharathi M, Sandhya C (2011) Isolation and characterization of candida species from oropharyngeal secretions of HIV positive individuals. N Dermatol Online 2: 119-124.

27. Taplin D, Zaias N, Rebell G, Blank H (1969) Isolation and recognition of dermatophytes on a new medium (DTM). Arch Dermatol 99: 203-209.

28. John $\mathrm{H}$, Barbara D, David Andies, Beth Arthington-skaggs, Brown SD, et al. (2008) M38-A2 Reference Method for Broth Dilution Antifungal Susceptibility Testing of Filamentous Fungi; Approved Standard - Second Edition 28:16.

29. Sastry YN, Padmaja IJ, Rao PR, Kirani KRLS, Kaladhar DSVGK, et al. (2012) In vitro dose dependent study on anti human pathogenic bacterial and free radical scavenging activities of methanolic seed coat extract of Borassus flabellifer I. Asian J Pharm Clin Res. 5: 83-86. 